\title{
Finite Element Model of Atlantoaxial Joint with Vertebral Artery and Simulation Traction Therapy
}

\author{
Changhua Lin, Hua Zhong, Huiyi Zhao, Shaoyin Duan ${ }^{1^{*}}$ \\ Zhongshan Hospital of Xiamen University, Xiamen 361004, China \\ *xmdsy@xmu.edu.cn \\ *The corresponding author
}

\begin{abstract}
This paper is to establish the finite element model (FEM) of atlantoaxial joint (AAJ) with vertebral artery (VA), and then have a clinical traction simulation, in order to observe the stress distribution and characteristics of AAJ and VA. CTA data of head and neck in 10 cases were used as the study objects from PACS database in our hospital, and they must be nothing abnormal detected with AAJ and VA, and with standard position and clear VA. The imaging data must be saved as DICOM, imported to Mimics and built the surface geometry model. Then it needed grids optimizing in Geomagic Studio and volume and element mesh in Hypermesh. In Abaqus, the meshed model would be given material assignments and form FEM of AAJ and VA. Finally, there are the simulations of AAJ movement and traction therapy, the results were showed in images and have an analysis and comparison. FEM of AAJ and VA had been established, there are 532921 nodes and 331309 elements. The simulation of rotary motion with the loads of $5 \mathrm{~N}, 2 \mathrm{~N}$ and $0.5 \mathrm{~N}$, the rotation angle of AAJ was $19.5^{\circ}, 8.9^{\circ}$ and $2.5^{\circ}$, the stress of posterior-arch of atlas was $3100-4200 \mathrm{~N} / \mathrm{mm} 2,500-1000 \mathrm{~N} / \mathrm{mm} 2$ and $200-400 \mathrm{~N} / \mathrm{mm} 2$, as similar as in the anterior-arch of atlas and VA. The simulation of traction therapy with loads of $5 \mathrm{~N}$ and $2 \mathrm{~N}$ on the flexion in $30^{\circ}, 15^{\circ}$, neutral position, extension in $15^{\circ}, 30^{\circ}$, the stress of posterior-arch of atlas was $6000-9000 \mathrm{~N} / \mathrm{mm} 2$, $1500-2500 \mathrm{~N} / \mathrm{mm} 2,2000-3000 \mathrm{~N} / \mathrm{mm} 2,4700-6500 \mathrm{~N} / \mathrm{mm} 2,5000-8000 \mathrm{~N} / \mathrm{mm} 2$ and 1500-3000 $\mathrm{N} / \mathrm{mm} 2,600-1300 \mathrm{~N} / \mathrm{mm} 2,500-1000 \mathrm{~N} / \mathrm{mm} 2,1600-2500 \mathrm{~N} / \mathrm{mm} 2,2000-3300 \mathrm{~N} / \mathrm{mm} 2$, as similar as in the nuchal ligament and pedicle of axis arch. FEM of AAJ with VA is in accordance with their anatomic structures and physiological function. In the motion simulation, the size of load is proportional to the motion angle and stress distribution, which is larger in the regions of VA, the anterior and posterior arch. In the traction simulation, the maximum stress is in the regions of nuchal ligament, posterior arch of atlas and axis pedicle in the position of extension $30^{\circ}$ and the minimum stress is in the neutral position $0^{\circ}$. The simulation results can guide the related parameters setting of traction therapy and may prevent the iatrogenic injury.
\end{abstract}

Keywords: Atlantoaxial joint with vertebral artery; Finite element model and simulation; Traction therapy

\section{富枢关节带椎动脉有限元建模与}

\section{牵引疗法模拟}

\author{
林长华，钟华，赵慧毅，段少银* \\ 厦门大学附属中山医院（福建省厦门市，361004） \\ *通讯作者 段少银 xmdsy@xmu.edu.cn
}

摘要：建立裹枢关节带椎动脉有限元模型，进行运动与牵引模拟，讨论裹枢关节，椎动脉区域的应力分布及受力特点。 我院 PACS 数据库头颈部血管造影(CTA)的数据中, 选择 10 例作为研究对象, 其体位标准, 㝘枢关节及双侧椎动脉未见明显 异常, 且图像清晰, 椎动脉显示清楚, 没有颈部其他疾病。以 DICOM 格式储存原始 CT 影像学数据, 将其导入 Mimics 软件 中建立面网格模型; 在 Geomagic Studio 软件中对面网格模型进行网格优化处理，然后将其导入 Hypermesh 中进行体网格及 
单元划分, 形成三维实体模型; 利用 Abaqus 软件进行解剖结构的材料赋值, 完成构建睘枢关节带椎动脉的有限元模型。最 后, 模拟不同载荷的睘枢关节运动与牵引疗法, 结果图像显示并进行分析与比较。建立裹枢关节带椎动脉有限元模型, 模型 由 532921 个节点, 331309 个单元构成。载荷 $0.5 \mathrm{~N}, 2 \mathrm{~N}, 5 \mathrm{~N}$ 模拟旋转运动时, 旋转角度分别为 $2.5^{\circ}, 8.9^{\circ}, 19.5^{\circ}$, 椎动脉后弓应 力值分别为 200-400 N/mm2, 500-1000 N/mm2, 3100-4200 N/mm2, 寰椎前弓, VA 区域与其相仿。模型载荷（ $5 \mathrm{~N}, 2 \mathrm{~N} ） 2 \mathrm{~N}, 5 \mathrm{~N}$ 牵引疗法时, 显示棘突, 睘椎后弓及枢椎椎弓根区域受力相仿, 睘椎后弓在前屈 $30^{\circ}, 15^{\circ}$, 中立位, 后伸 $15^{\circ}, 30^{\circ}$ 时应力值分 别为 $1500-3000 \mathrm{~N} / \mathrm{mm} 2,600-1300 \mathrm{~N} / \mathrm{mm} 2,500-1000 \mathrm{~N} / \mathrm{mm} 2,1600-2500 \mathrm{~N} / \mathrm{mm} 2,2000-3300 \mathrm{~N} / \mathrm{mm} 2$ 以及 $6000-9000$ N/mm2, 1500-2500 N/mm2, 2000-3000 N/mm2, 4700-6500 N/mm2, 5000-8000 N/mm2。结论：寰枢关节附椎动脉有限元模型与解剖一 致, 模拟运动符合生理功能; 载荷大小与褱枢关节受力, 运动角度成正比, 旋转运动曼椎前, 后弓及 VA 区域受力最大。牵 引模拟发现项韧带, 裹椎后弓及枢椎椎弓根区域在后伸 $30^{\circ}$ 受力最大，而中立位 $0^{\circ}$ 受力最小; 模拟结果有利于牵引疗法相关 参数的设置, 防止医源性损伤。

关键词: 寰枢关节; 椎动脉; 有限元建模; 应用模拟

\section{前言}

寰枢关节区结构复杂，周围结构血管，神经功能关系密切，疾病危害性大，相关的解剖与疾病研究是 目前研究的热点 ${ }^{[1-5]}$ 。本研究采用螺旋 CT 实体扫描数据, 建立畺枢关节带椎动脉的有限元模型, 对该模型 进行了验证, 并进行了牵引疗法的模拟实验。通过加载不同的车引力及帚引角度, 研究睘枢关节各部分生 物力学的分布情况, 以期在提供原始模型的基础上, 为临床牵引治疗等治疗方案提供生物力学理论依据 ${ }^{[6-9]}$ 。

\section{1. 实验与方法}

\section{1. 建模与软件}

1.1. 有限元分析是利用数学近似的方法对真实物理系统（几何和载荷工况）进行模拟。还利用简单而 又相互作用的元素, 即单元, 就可以用有限数量的未知量去逼近无限未知量的真实系统。实现用较简单的 问题代替复杂问题后再求解，过程分三个阶段：前处理，加载计算，后处理。

1.1.2 本研究实现寰枢关节附椎动脉有限元建模流程如下: 1. 获得 DICOM 格式 CT 图像; 2. Mimics10.0 软 件中建立寰枢关节附椎动脉表面几何模型；3. Geomagic Studio 软件中对表面几何模型进行优化处理; 4. Hypermesh 软件中进行体网格及单元划分, 并确定单元类型; 5. Abaqus 软件中进行模型装配及材料赋值, 生成寰枢关节附椎动脉有限元模型; 6. Abaqus 软件对生成的有限元模型定义接触, 设定边界条件, 施加载 荷 Abaqus 软件中进行后处理, 整理计算结果

\section{2 有限元模型的建立}

1.2.1 从我院 PACS 数据库头颈部血管造影 (CTA) 的数据中, 选择 10 例作为研究对象, 其体位标准, 睘枢 关节及双侧椎动脉未见明显异常, 且图像清晰, 椎动脉显示清楚, 没有颈部其他疾病。以 DICOM 格式储存 原始 CT 影像学数据。

1.2.2 扫描条件: 扫描管电压 $120 \mathrm{KV}$, 管电流 $600-700 \mathrm{~mA}$, 准直宽度 $0.625 \mathrm{~mm}$, 螺距 0.984 , 旋转时间 $330 \mathrm{~ms} / \mathrm{rot}$ 。扫描方法: 使用双筒高压注射器经前臂静脉流率 $3.5 \mathrm{ml} / \mathrm{s}$ 注入 A 筒 $40 \mathrm{ml}$ 碘普罗胺 $370 \mathrm{mg} \mathrm{I} / \mathrm{ml}$, 随后 B 筒追加生理盐水 $30 \mathrm{~m} 1$ 。

1.2.3 实体模型建立与优化:Mimics 软件中建立寰枢关节附椎动脉表面几何模型; Geomagic Studio 软件 中对表面几何模型进行消除噪声, 光滑等优化处理, 优化后的表面几何模型; 在 Hypermesh 软件 3D 模块 中, 进行体网格及单元划分, 定义单元类型为修正后 10 节点的二阶四面体单元（C3D10M）类型。成功划 
分体网格后, 所得模型包括新生成体网格和未划分时面网格, 然后进行较低级面网格删除并以. INP 格式输 出备用。

1.2. 4 有限元模型的建立及材料属性定义:本研究的材料属性相关数值参考相关文献报 [6-8] 的实验结果 （表 1）。骨性结构统一采用各向同性的弹性材料属性, 分别设杨氏弹性模量, 泊松比, 密度为 $12000 \mathrm{Mpa}$, $0.3,1.83 \times 10^{-9} \mathrm{t} / \mathrm{mm} 3$ 。㓞带包括: 十字韧带，翼状韧带，尖韧带，前纵韧带，后纵韧带以及项韧带 ${ }^{[6,11]}$ 。

表 1. 颈椎各结构的材料属性

\begin{tabular}{cccc}
\hline 材料属性 & 弹性模量 $(\mathrm{MPa})$ & 泊松比 & 密度 $\left(\times 10^{-9} \mathrm{t} / \mathrm{mm}^{3}\right)$ \\
\hline 骨性结构 & 12000 & 0.30 & 1.83 \\
椎动脉 & 1 & 0.49 & 1.10 \\
十字韧带 & 20 & 0.40 & 1.10 \\
项㓞带 & 20 & 0.40 & 1.10 \\
前, 后纵韧带 & 10 & 0.40 & 1.10 \\
翼状㓞带 & 5 & 0.40 & 1.10 \\
尖㓞带 & 5 & 0.40 & 1.10 \\
\hline
\end{tabular}

1.2 .5 定义接触, 设定边界条件: 睘枢关节包括正中寰齿关节, 睘枢外侧关节, 定义为滑动接触; 韧带, 骨性结构附着点定义为捆绑约束。限定边界条件时, 需对建立的有限元模型第 3 颈椎椎体及附件下 $1 / 3$ 限 定为固定, 即将各节点的各向自由度均设置为 “ 0 ” ${ }^{[10,11]}$ 。

\section{2 结果}

\section{1 模型的建立及验证}

2. 1.1 模型的建立: 成功建立寰枢关节附椎动脉有限元模型, 由 532921 个节点, 331309 个单元构成。该 模型由两部分组成：骨性结构和软组织结构。骨性结构部分包括㐿底骨，睘椎，枢椎以及第 3 颈椎; 软组 织部分包括十字韧带，尖韧带，翼状韧带，前纵韧带，后纵韧带，项韧带及椎动脉。

2.1 .2 模型在不同载荷状态下的右侧旋转运动模型分别在 $0.5 \mathrm{~N}, 2 \mathrm{~N}, 5 \mathrm{~N}$ 载荷下模拟右侧旋转运动, 旋转 角度依次为: $2.5^{\circ}, 8.9^{\circ}, 19.5^{\circ}$ （图 1)，由图形的形变大小可看出模型的形变大小与载荷大小成正 相关关系。

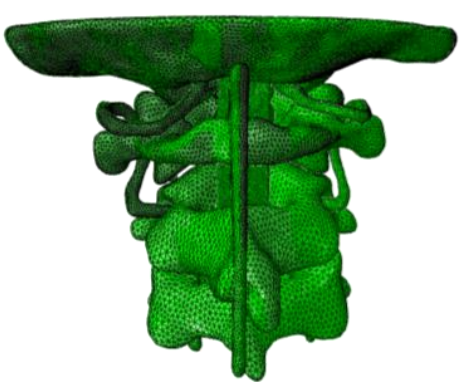

a

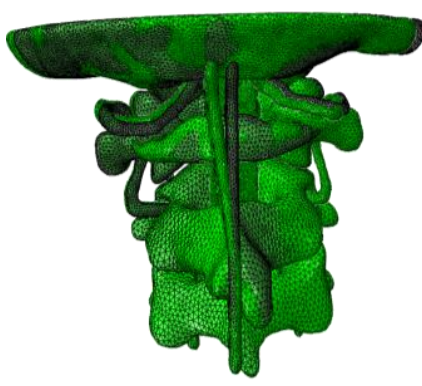

b

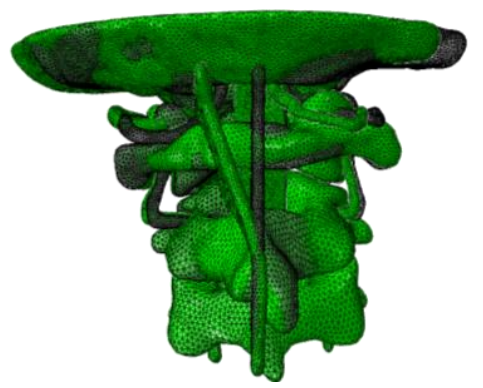

c

图 1: a, b, c 分别为模型在 $0.5 \mathrm{~N}, 2 \mathrm{~N}, 5 \mathrm{~N}$ 载荷下右侧旋转运动形变前后融合图像: 黑色为形变前模型, 绿色为形变后模型。 
2.1.2 模型在不同载荷状态下最大应力分布情况(表 2, 图 2)

表 2 寰枢关节不同载荷下旋转运动的应力分布情况 $\left(\mathrm{N} / \mathrm{mm}^{2}\right)$

\begin{tabular}{cccc}
\hline 载荷大小 & $0.5 \mathrm{~N}$ & $2 \mathrm{~N}$ & $5 \mathrm{~N}$ \\
\hline 寰椎前弓 & $150-400$ & $500-900$ & $3300-4300$ \\
寰椎前弓 & $200-400$ & $500-1000$ & $3100-4200$ \\
椎动脉 & $150-350$ & $450-800$ & $3000-4000$ \\
枢椎齿突 & $50-100$ & $150-300$ & $300-600$ \\
枢椎椎弓根 & $25-50$ & $100-250$ & $300-400$ \\
\hline
\end{tabular}

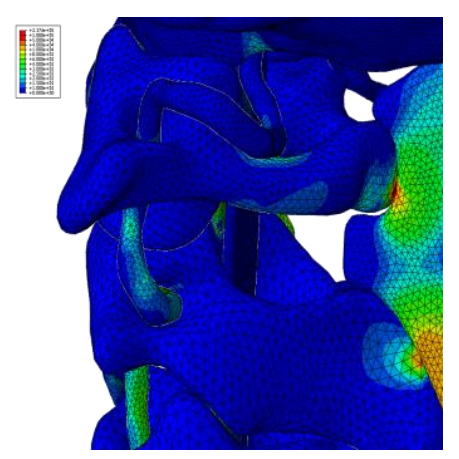

a

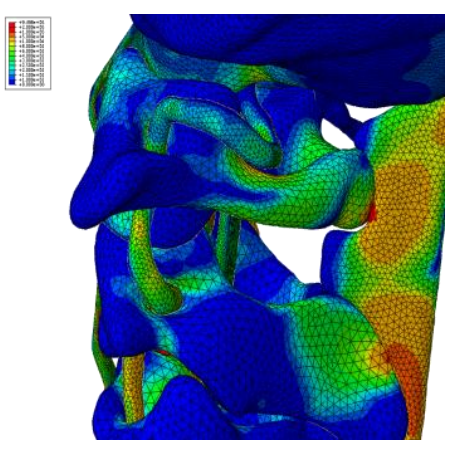

b

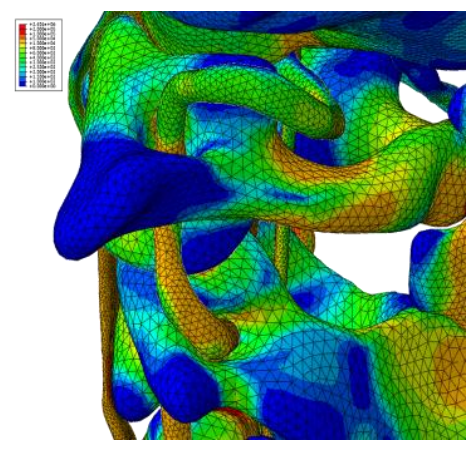

c

图 2:a, b, c 分别为模型在 $5 \mathrm{~N}, 2 \mathrm{~N}, 0.5 \mathrm{~N}$ 载荷状态下的应力云图

2.1.3 不同载荷状态寰枢关节面的位移情况：实验选择寰椎外侧关节面前缘第 874 号节点为代表，计算其 位移情况，并绘制位移-时间曲线图（图 3），模型在 $0.5 \mathrm{~N}, 2 \mathrm{~N}, 5 \mathrm{~N}$ 载荷状态下睘椎外侧关节面第 874 号节 点右侧旋转运动的位移分别为 $2.2 \mathrm{~mm}, 8.9 \mathrm{~mm}, 22.1 \mathrm{~mm}$ 。

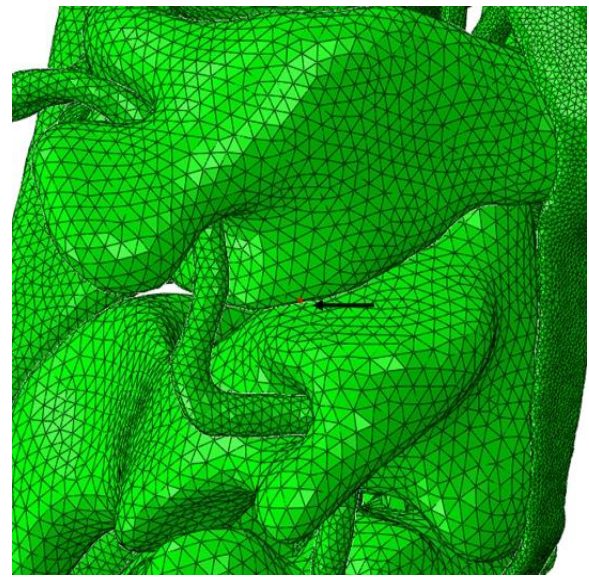

a

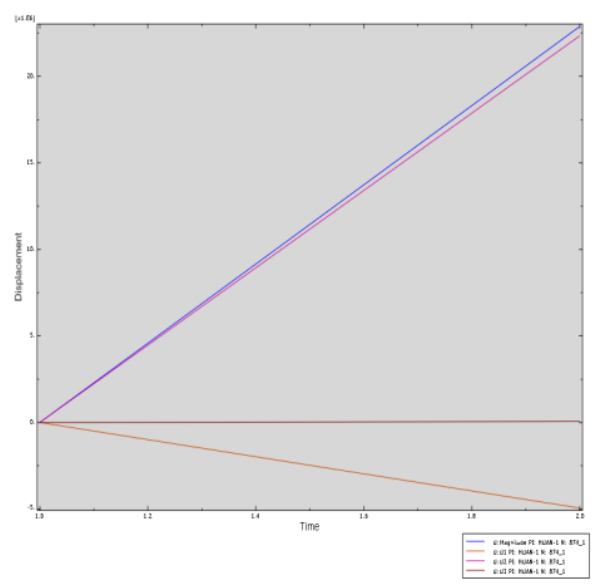

$\mathrm{b}$

图 $3: a$ 显示寰椎外侧关节面前缘上第 874 号节点位置; $b$ 为模型牵引过程第 874 号节点位移一时间曲线图。

\section{2 寰枢关节牵引模拟显示受力分布}

2.2.1 利用已经建立的睘枢关节有限元模型模拟正常人在平卧位时分别施以不同荷载，不同角度的牵引 疗法, 观察模型各部位的受力分布情况（图 4,5）。 
2.2.2 牵引疗法模拟后寰枢椎各部分结构的应力大小分布情况（表 3,4）。

表 3 寰枢关节 $5 \mathrm{~N}$ 载荷牵引疗法模拟的应力分布情况 $(\mathrm{N} / \mathrm{mm} 2)$

\begin{tabular}{lccccc}
\hline 部位 & 寰椎前弓 & 睘椎后弓 & 椎动脉 & 枢椎齿突 & 枢椎椎弓根 \\
\hline 后伸 $30^{\circ}$ & $900-1200$ & $5000-8000$ & $300-900$ & $1200-1700$ & $4500-7000$ \\
后伸 $15^{\circ}$ & $800-1100$ & $4700-6500$ & $200-800$ & $200-700$ & $3500-4800$ \\
中立位 $0^{\circ}$ & $600-1200$ & $2000-3000$ & $150-600$ & $1200-1800$ & $1100-1600$ \\
前屈 $15^{\circ}$ & $1000-2000$ & $1500-2500$ & $400-1000$ & $1800-3000$ & $1200-2000$ \\
前屈 $30^{\circ}$ & $2500-4000$ & $6000-9000$ & $500-2000$ & $4000-7000$ & $5500-7000$ \\
\hline
\end{tabular}

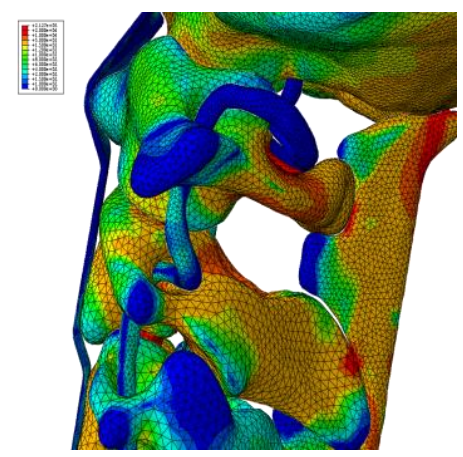

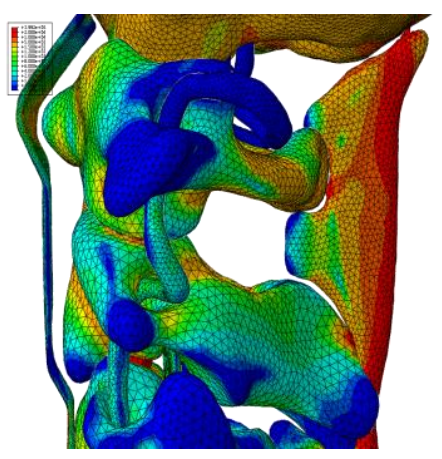

b

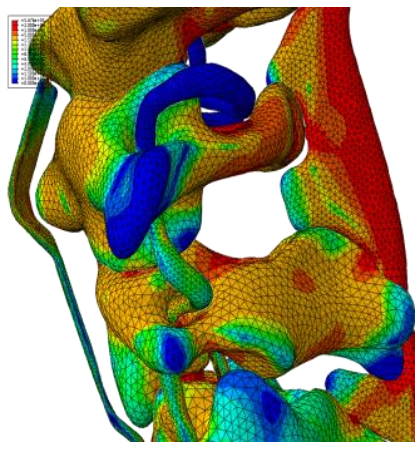

c

图 4: a, b,c 为模型在 $5 \mathrm{~N}$ 牵引力分别后伸 $30^{\circ}$, 中立位 $0^{\circ}$, 前屈 $30^{\circ}$ 的应力云图。

表 4 寰枢关节结构 $2 \mathrm{~N}$ 载荷牵引模拟应力分布 $(\mathrm{N} / \mathrm{mm} 2)$

\begin{tabular}{cccccc}
\hline 部位 & 寰椎前弓 & 睘椎后弓 & 椎动脉 & 枢椎齿突 & 枢椎椎弓根 \\
\hline 后伸 $30^{\circ}$ & $300-700$ & $2000-3300$ & $100-300$ & $200-700$ & $1800-2800$ \\
后伸 $15^{\circ}$ & $200-600$ & $1600-2500$ & $50-200$ & $150-500$ & $1300-2000$ \\
中立位 $0^{\circ}$ & $400-700$ & $500-1000$ & $200-400$ & $500-800$ & $400-700$ \\
前屈 $15^{\circ}$ & $500-800$ & $600-1300$ & $300-500$ & $700-1200$ & $500-800$ \\
前屈 $30^{\circ}$ & $600-1000$ & $1500-3000$ & $400-600$ & $1300-1800$ & $1200-2000$ \\
\hline
\end{tabular}

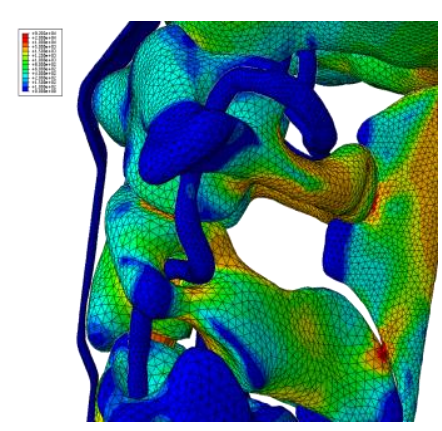

a

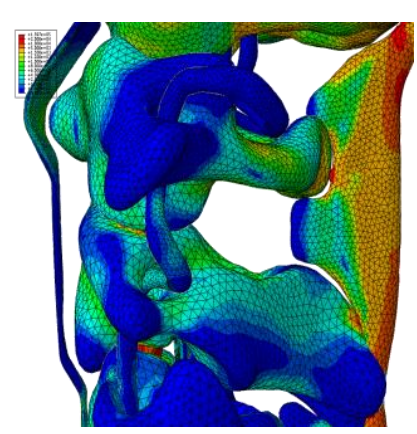

b

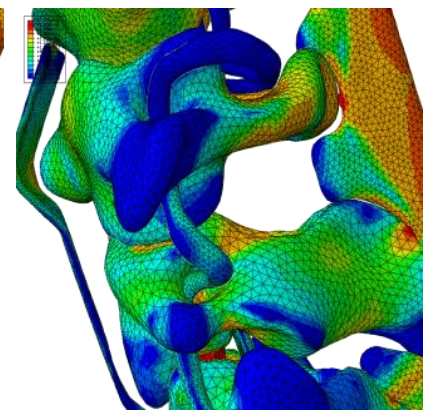

$\mathrm{c}$

图 5:a, b,c 为模型在 $2 \mathrm{~N}$ 牵引力分别后伸 $30^{\circ}$, 中立位 $0^{\circ}$, 前屈 $30^{\circ}$ 的应力云图。 


\section{3 讨论}

3.1 生物力学有限元分析法的模型验证: 目前有限元孙玛等 ${ }^{[10]}$ 认为以往有限元模型的实验数据的验证是基 于退变或老化的标本, 这样的实验数据并不太准确, 且不同的学者在验证模型时因采用不同的加载条件和 边界条件, 所得的结果往往也不尽相同。因为模型极度简化了真实复杂的人体结构, 模型在实验中的各种 状况也是对人体运动时各肌群间相互作用的复杂过程的极度简化。简单地给予单个方向的载荷或多个方向 力的合力载荷, 其计算结果与实体结果仍有相当差距, 所以即使在实验中证明模型有效, 也并不表示模型 能复制人体在复杂的载荷条件下的实际结果。因此, 在进行有限元模型的模拟研究中, 对于模型的材料属 性, 接触属性等关键参数仍需要进行更加深入的研究, 以使模型更接近真实的人体结构解剖特点与临床要 求 $^{[12]}$ 。

3.2 有限元模型模拟的精确性: 在过去的有限元模拟研究中, 大部分学者认为模型的应力产生于位移, 位 移的改变相较于应力更加准确, 且认为模型对位移变化更敏感, 少有研究对模型中应力集中的精确值进行 详尽计算, 研究主要集中于对施加载荷计算后模型变形前后产生的形变进行对比, 以确定模型的有效性, 主要用于对模型进行定性分析, 而没有分析模型内应力集中的定量值。本研究运用 Abaqus 有限元软件进 行研究, 虽可确切测得模型应力集中区域受力的范围值, 具有较好的可比性。但还不能准确包括活体内的 各种复杂情况变化, 其有效性及确切性还应持谨慎态度, 实验研究所得结果仅供临床参考与研究探讨 ${ }^{[12]}$ 。

3.3 牵引重量的影响: 杨利学等作者 ${ }^{[13]}$ 认为, 与传统的牵引重量相比, 较大重量的牵引更符合人体生物力 学原理特性, 更有利于神经根型颈椎病的康复治疗。陈庆法等 ${ }^{[14]}$ 认为首次牵引重量应从 5.0-6.0 kg 开始, 而后根据患者的耐受程度再逐渐增加牵引重量。并可根据患者的不同体重选择不同的牵引重量进行颈椎牵 引, 否则牵引重量过大可能反而加重病情, 甚至在牵引治疗时即可产生严重的不适感, 而牵引重量若过小 则可能达不到治疗效果。本实验研究证实: 在甡引角度一定时, 实验所测得的睘枢关节各部位应力值的大 小与甡引重量的大小呈正相关关系, 局部应力的变化随着牵引重量的增大而增大, 说明牵引的作用与重量 直接相关 ${ }^{[15]}$ 。

3.4 牵引角度的影响: 李勇等作者 ${ }^{[16]}$ 认为, 在亜引角度控制仪控制下, 在中立位, 前屈 $10^{\circ}$, 后伸 $10^{\circ}$ 分别进行牵引治疗, 结果显示中立位时对椎体的纵向牵拉作用最大, 而前屈牵引治疗时应力主要集中于椎 体的后缘, 后伸牵引治疗时应力则主要集中在位于椎体后部的小关节。有部分学者根据不同类型颈椎病分 别予以不同角度的牵引治疗。张锦明等 ${ }^{[17]}$ 认为: 神经根型颈椎病适合采用前屈 $20^{\circ}-30^{\circ}$ 进行牵引, 脊髓 型则以后伸 $10^{\circ}-15^{\circ}$ 为宜, 椎动脉型以前屈 $5^{\circ}$ 以下为最佳。本实验研究证实: 在牵引力一定时, 实验 结果所测得的寰枢关节各部位应力值大小与牵引角度呈正相关关系, 中立位时各部位的应力值最小, 前屈 或后伸位时应力值随着牵引角度的增大而增大, 说明牵引角度的改变与局部受力的大小有关。

3.5 牵引疗法影响因素: 不同载荷牵引模拟的应力分布云图与应力测量值, 显示当牵引角度一定时, 寰枢 关节各部位的应力值随牵引力的增大而增大; 当牵引力一定时, 睘枢关节各部位的应力值随牵角度的增大 而增大; 在各车引力及角度情况下寰椎后弓均可见应力明显集中现象, 项韧带, 睘椎后弓与两侧块连接部 应力集中尤为明显, 且随珲引重量及角度增加而应力值增大; 牵引时牵引角度为前屈或后伸, 睘椎前弓及 枢椎椎弓根应力值随牵引角度增加而增加; 枢椎齿突前屈牵引时应力集中较明显, 且随不论前屈或后伸牵 引应力值均随甡引角度增大而增大。 
3.6 兾椎后弓骨折的生物力学: 寰椎结构的特殊性包括: 没有椎体, 棘突以及关节突, 而是由前, 后弓, 侧 块及横突围成的环形结构, 两侧块与前, 后弓相连接的部位是睘椎较为薄弱部位, 是骨折的好发部位。睘 椎是连接头夰与脊柱间的力量传递的交界结构, 由于睘椎侧块为外厚, 内薄楔形结构, 所以在力传递的过 程中, 会产生较大的外分力并集中暴发于两侧块与前，后弓连接部易发生骨折 ${ }^{[18]}$ 。本实验所建模型模拟牵 引发现项韧带，寰椎后弓，枢椎椎弓根应力较为集中，尤其为后弓与两侧块连接部相当于椎动脉沟处应力 集中现象明显，与上述观点相符，并可能影响椎动脉受力与血流。

\section{4. 结语}

睘枢关节带椎动脉有限元模型符合正常运动规律，载荷大小与寰枢关节受力，运动角度成正比; 明确 牽引目的情况下, 可以提前模拟牵引的效果, 减少盲目的牵引参数设置与尝试。睘枢关节牵引模拟显示项 韧带, 寰椎后弓, 枢椎椎弓根应力较为集中, 后弓与两侧块连接部为寰枢关节薄弱部位, 临床牵引疗法时 应考虑牵引角度，挂掉重量等，以防止受力过大而导致医疗损伤。

\section{5. 致谢}

厦门市科技计划项目（3502Z20144025）。

\section{Acknowledgement}

Science and technology program of Xiamen (3502Z20144025).

\section{参考文献}

[1] Lv S, He H, Yang L, et al. Experimental study on the atlanto-axial joint and related structures with regional anatomy and medical imaging [J]. West Indian Med J. 2011, 60 (5):548-52.

[2] Nakamura N, Inaba Y, Aota $\mathrm{Y}$, et al. New radiological parameters for the assessment of atlantoaxial instability in children with Down syndrome: the normal values and the risk of spinal cord injury[J]. Bone Joint J. 2016, 98-B (12):1704-1710.

[3] Niknejad HR, van Calenbergh F, Demaere1 P, et al. Accessory atlantoaxial ligament avulsion fracture of the axis: Are there any clinical implications? [J]. J Craniovertebr Junction Spine. 2016, 7(4):273-275.

[4] Jhawar SS, Nunez M, Pacca P, et al. Craniovertebral junction $360^{\circ}$ : A combined microscopic and endoscopic anatomical study [J]. J Craniovertebr Junction Spine, 2016, 7(4):204-216.

[5] Komatsu K, Ozaki A, Iwasaki K, et al. Bilateral Vertebral Artery Aneurysms at the Atlantoaxial Joint Level Causing Recurrent Stroke. Intern Med. 2016, 55(22):3365-3368.

[6] 刘治华, 许伟超, 张新民, 等 颈椎 $\mathrm{C} 2 \sim 7$ 三维有限元模型的建立与最优角度牵引仿真研究 $[\mathrm{J}]$. 郑州大学学报(医学 版)，2016，51(3)：359-363.

[7] Hu Y, Dong WX, Hann S, et al. Construction of Finite Element Model for an Artificial Atlanto-Odontoid Joint Replacement and Analysis of Its Biomechanical Properties [J]. Turk Neurosurg. 2016, 26(3):430-436.

[8] Zang Q, Liu Y, Wang D, et al. An Experimental Biomechanical Study on Artificial Atlanto-odontoid Joint Replacement in Dogs [J]. Clin Spine Surg. 2016 Jul 14. [Epub ahead of print] PubMed PMID: 27429156.

[9] Wei G, Shi C, Wang Z, et al. Surgical Outcome and Prognostic Analysis of Transoral Atlantoaxial Reduction Plate System for Basilar Invagination: A Voxe1-Based Morphometry Study[J]. J Bone Joint Surg Am. 2016, $98(20): 1729-1734$.

[10] 孙玛, 王诗成, 杨水泉, 等. 正常人体下颈椎有限元模型的建立及验证 [J]. 中国康复理论与实践. 2013, $29(7): 631-634$. 
[11] 张冠军, 魏嵬, 曹立波, 等., 3 岁儿童 C4-C5 颈椎有限元建模方法研究 $[J]$. 湖南大学学报 (自然科学版), 2016, $43(2): 8-14$.

[12] Zhang BC, Liu HB, Cai XH, et al. Biomechanical comparison of a novel transoral atlantoaxial anchored cage with established fixation technique - a finite element analysis [J]. BMC Musculoskelet Disord. 2015, 16(9) : $261-271$.

[13] 杨利学, 酒涛, 刘智斌, 等. 角度牵引下不同时间与重量对颈椎病疗效影响的临床研究 [J]. 现代中医药, 2009, $29(3): 5-7$.

[14] 陈庆法. 间歇牵引并超声调制中频电流治疗神经根型颈椎病的观查 [J]. 中华物理医学与康复杂 志, 2002, $24(9): 558$.

[15] 黄俊杰, 间晓东, 孙宏慧, 等. 枕领带牵引辅以头颈胸支具外固定治疗儿童寰枢关节半脱位 31 例 $[J]$. 昆明医科大学 学报, 2015, 36(3):60-62.

[16] 李勇, 李振宇, 鲁尧, 等. 不同角度牵引治疗颈椎病的力学效果分析研究 [J]. 中国中医骨伤科杂志, 2008, 16 (9) : $41-42$.

[17］张锦明, 陆唱, 卢宇. 颈椎牵引角度对神经根型颈椎病疗效的影响 [J]. 现代康复, 1999, 3(12)：1484.

[18] Mead LB, Millhouse PW, Krystal J, et al. C1 fractures: a review of diagnoses, management options, and outcomes [J]. Curr Rev Musculoskelet Med. 2016, $9(3): 255-262$.

\section{References}

[1] Lv S, He H, Yang L, et al. Experimental study on the atlanto-axial joint and related structures with regional anatomy and medical imaging [J]. West Indian Med J. 2011, 60(5):548-52.

[2] Nakamura N, Inaba Y, Aota $\mathrm{Y}$, et al. New radiological parameters for the assessment of atlantoaxial instability in children with Down syndrome: the normal values and the risk of spinal cord injury[J]. Bone Joint J. 2016, 98-B (12):1704-1710.

[3] Niknejad HR, van Calenbergh F, Demaerel P, et al. Accessory atlantoaxial ligament avulsion fracture of the axis: Are there any clinical implications? [J]. J Craniovertebr Junction Spine. 2016, 7(4):273-275.

[4] Jhawar SS, Nunez M, Pacca P, et al. Craniovertebral junction 360: A combined microscopic and endoscopic anatomical study [J]. J Craniovertebr Junction Spine, 2016, 7(4):204-216.

[5] Komatsu K, Ozaki A, Iwasaki K, et al. Bilateral Vertebral Artery Aneurysms at the Atlantoaxial Joint Level Causing Recurrent Stroke. Intern Med. 2016, 55(22):3365-3368.

[6] Liu Zhihua, Xu Weichao, Zhang Xinmin, et al. A Simulation Study of 3D Finite Element Modeling of C2 7 Cervical Vertebra C2 7 and the Traction Treatment of Optimal Angle[J]. Journal of Zhengzhou University (Medical Sciences), 2016, 51(3): 359-363.

[7] $\mathrm{Hu} \mathrm{Y,} \mathrm{Dong} \mathrm{WX,} \mathrm{Hann} \mathrm{S,} \mathrm{et} \mathrm{al.} \mathrm{Construction} \mathrm{of} \mathrm{Finite} \mathrm{Element} \mathrm{Model} \mathrm{for} \mathrm{an} \mathrm{Artificial}$ Atlanto-Odontoid Joint Replacement and Analysis of Its Biomechanical Properties [J]. Turk Neurosurg. 2016, 26(3):430-436.

[8] Zang Q, Liu Y, Wang D, et al. An Experimental Biomechanical Study on Artificial Atlanto-odontoid Joint Replacement in Dogs [J]. Clin Spine Surg. 2016 Jul 14. [Epub ahead of print] PubMed PMID: 27429156.

[9] Wei G, Shi C, Wang Z, et al. Surgical Outcome and Prognostic Analysis of Transoral Atlantoaxial Reduction Plate System for Basilar Invagination: A Voxel-Based Morphometry Study[J]. J Bone Joint Surg Am. 2016, 98(20):1729-1734. 
[10] Sun Yu, Wang Shicheng, Yang Shuiquan, et al. Establishment and Verification of Finite Element Model of Cervical Vertebra of Normal People[J]. Chinese Journal of Rehabilitation Theory and Practice. 2013, 29(7): 631-634.

[11]Zhang Guanjun, Wei Wei, Cao Libo, et al. A Research of Finite Element Modeling Method of C4-C5 Cervical Vertebra of 3-year-old Children. Journal of Hunan University(Natural Sciences), 2016,43(2): 8-14.

[12]Zhang BC, Liu HB, Cai XH, et al. Biomechanical comparison of a novel transoral atlantoaxial anchored cage with established fixation technique - a finite element analysis [J]. BMC Musculoskelet Disord. 2015, 16(9): 261-271.

[13] Yang Lixue, Jiu Tao, Liu Zhibin, etc. Effect of Different Time and Weight to Curative Effect of Cervical Spondylosis under Perspective Traction [J].Modern Traditional Chinese Medicine,2009,29(3):5-7.

[14] Chen Qingfa. Observation on Intermittent Traction and Ultrasound-Modulated Medium Frequency Current to Treat Radiculopathy Cervical Spondylosis [J].Chinese Journal of Physical Medicine and Rehabilitation,2002,24(9):558.

[15] Huang Junjie, Yan Xiaodong, Sun Honghui,etc.31 Cases of Occipital Jaw Band Traction and Neck Chest Brace External Fixation to Heal Children Atlantoaxial Subluxation [J].Journal of Kunming Medical University,2015,36(3):60-62.

[16] Li Yong, Li Zhenyu, Lu Yao,etc. Analytical Investigation of Mechanical Effects for Treating Cervical Spondylopathy under Different Angles Traction [J].Chinese Journal of Traditional Medical Traumatology \& Orthopedics,2008,16(9):41-42.

[17]Zhang Jinming, Lu Chang, Lu Yu. Curative Effect of Cervical Traction Angle to Radiculopathy Cervical Spondylosis [J].Modern Rehabilitation, 1999,3(12):1484.

[18] Mead LB, Millhouse PW, Krystal J, et al. C1 fractures: a review of diagnoses, management options, and outcomes [J]. Curr Rev Musculoskelet Med. 2016, 9(3):255-262. 